\title{
Descriptive statistical analysis based on patients' EEG energy in coma and quasi-brain-death state
}

\author{
Yao $\mathrm{Miao}^{(1)}$ and Jianting $\mathrm{Cao}^{(2)}$ \\ ${ }^{(1)}$ Graduate School of Engineering, Saitama Institute of Technology, Japan \\ e6006gxp@sit.ac.jp \\ ${ }^{(2)}$ Graduate School of Engineering, Saitama Institute of Technology, Japan \\ cao@sit.ac.jp
}

\begin{abstract}
In this paper, measure of descriptive statistical analysis based on EEG (electroencephalography) energy was proposed to evaluate coma and quasi-brain-death patients' EEG. Firstly, 36 cases of EEG (coma: 19; quasibrain-death: 17) were analyzed. Specifically, 60s EEG data of each case were randomly selected and processed to get energy data by Dynamic 2T-EMD (turning tangent empirical mode decomposition), then energy data for 36 cases of EEG obtained were grouped and analyzed by using descriptive statistical analysis, and finally results were displayed by visual method. Results show significantly that energy for coma patients' EEG is higher than that for quasi-brain-death patients' EEG. And the EEG energy data set for quasi-braindeath is relatively more concentrate.
\end{abstract}

\section{Keywords}

Descriptive statistical analysis, EEG energy, Brain death.

\section{Subject Classification}

Signal processing

\section{Type (Method/Approach)}

Algorithm analysis; Experimentation; EEG

\section{Introduction}

In BDD (brain death determination) of many countries, EEG has become an important metric. There were some measures developed to denoise and feature extraction for EEG in coma and brain-death state. For example, ICA (independent component analysis) [1], several complexity measures [2], EMD [3, 4], Dynamic 2T-EMD [5] which is the optimization based on 2T-EMD [6] were applied to process coma and brain-death patients' EEG. Since BDD is a serious issue, it is necessary to develop different methods from different perspectives to improve the accuracy and credibility of results.

In this paper, we proposed descriptive statistical analysis based on patients' EEG energy to evaluate coma and quasi-brain-death patients' EEG. There were two parts of work included in the paper. The first part focused on the method proposed in this research, which was visual statistical analysis based on EEG energy. And here EEG energy were computed by using Dynamic 2T-EMD. The second part explained the experiment process. In this part we conducted a visual statistical analysis to the energy results processed by Dynamic 2TEMD. Specifically, we selected randomly 60s EEG data from 19 cases of EEG in coma state and 17 cases of EEG in quasi-brain-death state to process by using Dynamic 2T-EMD algorithm firstly. And energy results obtained were grouped from three aspects, that were different cases, different channels, different state separately. And then the EEG energy after grouping were analyzed by applying descriptive statistical analysis. At last statistical results were visualized. Results show that energy for coma patients' EEG is obviously higher than that for quasi-brain-death patients' EEG. And the EEG energy in coma state is higher than $1.00 \times 10^{4}$, while EEG energy in quasi-brain-death state is lower than $1.00 \times 10^{4}$. Furthermore, the EEG energy data set for coma group are relatively more discrete. This will be useful to provide the physicians with more accurate and reliability visualized references.

\section{Materials and Methods}

EEG data. There were 36 cases of coma and quasi-brain-death patients' EEG with a total of 19 cases of EEG in coma state and 17 cases of EEG in quasi-brain-death were analyzed, which were came from a Chinese hospital from June 2004 to March 2006 and the EEG recording got the permission of the patients' families [7]. For each case of patient's EEG, there were 6 electrodes of EEG were recorded, respectively Fp1, $\mathrm{Fp} 2, \mathrm{~F} 3, \mathrm{~F} 4, \mathrm{~F} 7$ and F8. It is noted that 5 electrodes of EEG that came from 3 cases of EEG in coma state were invalid. 
Descriptive Statistical analysis. Since the EEG data were recorded in the ICU, which were mixed with complex noise, statistical analysis visual analysis were used to analyze the energy data processed by Dynamic 2T-EMD to improve the accuracy and reliability of results. In this paper, we focused on visual descriptive statistical analysis method. Descriptive statistics are summary statistics that quantitatively describe or summarize features of a collection of information [8]. Here basic measures of descriptive statistics including measure of central tendency and measure of dispersion were applied to evaluate dynamic energy data obtained, in which mean and median were used to describe the central tendency of data set, and standard deviation and percentile were introduced to evaluate the dispersion of data set.

(a) Mean and Median line for 36 cases

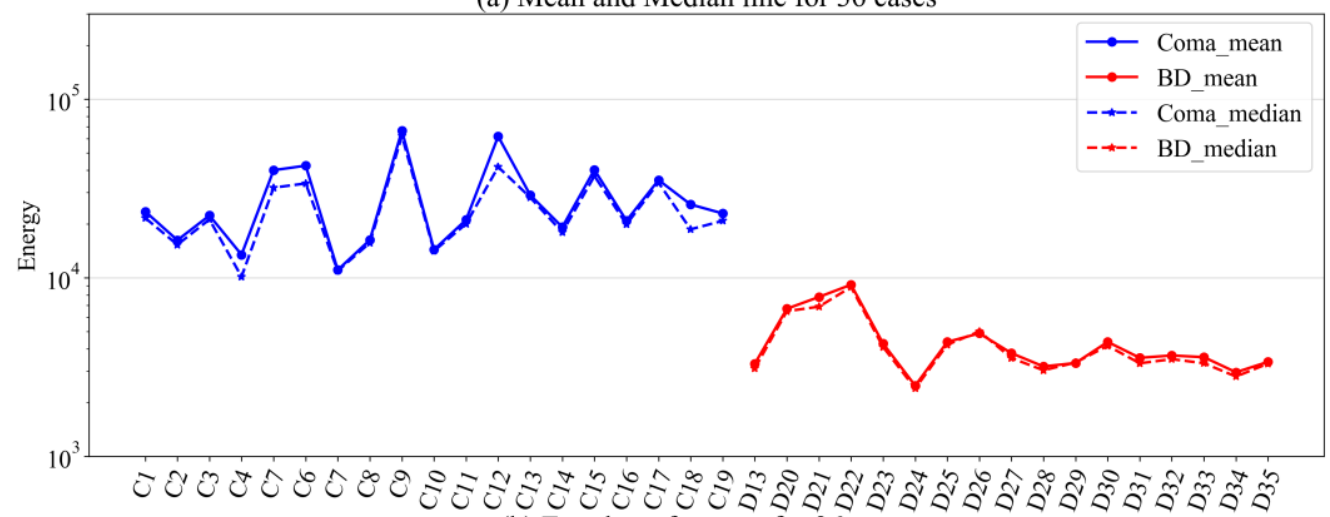

(b) Errorbar of energy for 36 cases

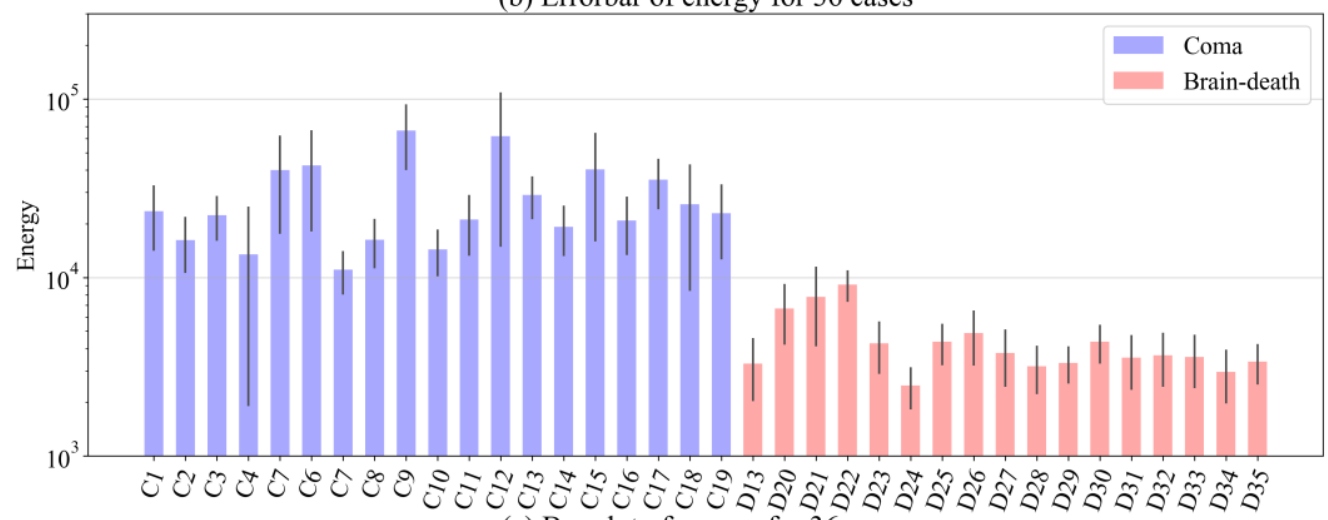

(c) Boxplot of energy for 36 cases

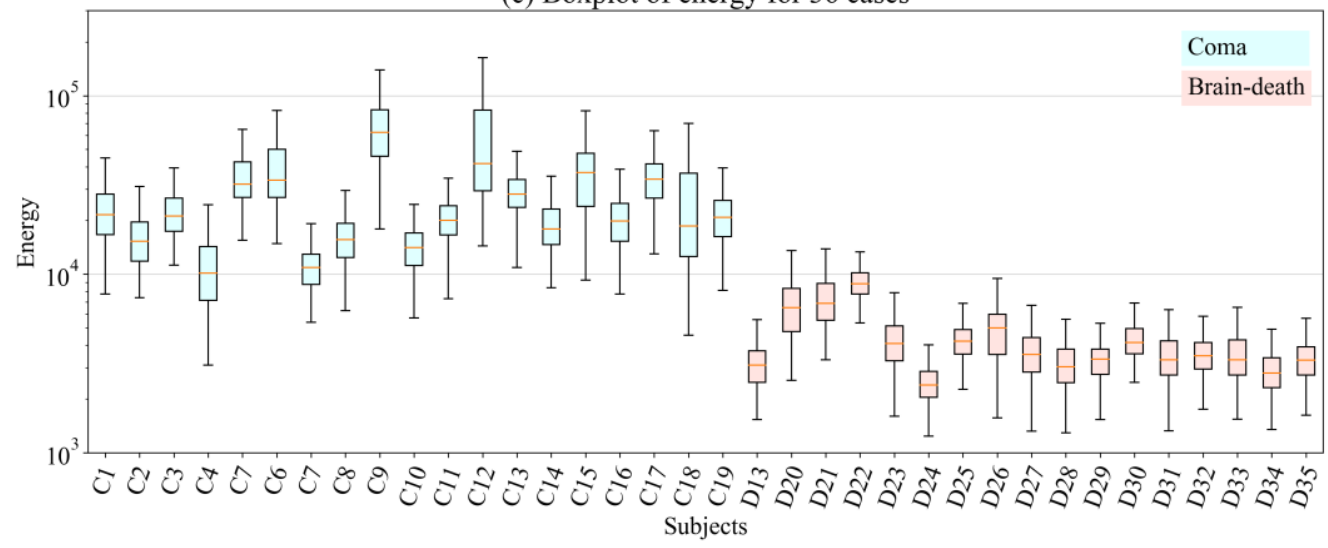

Figure 1. The statistical analysis results of EEG energy for 36 coma and quasi-brain-death EEG for average channel.

Experiment measures. Specifically, there were 4 steps to conduct the experiment measure:

1) Select randomly 60s EEG data from 36 cases of coma and quasi-brain-death patients' EEG (coma: 19; quasi-brain-death: 17). Here there were 5 channels of EEG data with values equal 0 from 3 cases invalid.

2) Process these EEG data by applying Dynamic $2 T$-EMD algorithm and 211 time-energy series (36 cases $\times 6$ channels - 5 invalid series) were obtained.

3) Group the 211 time-series according to 3 ways:

(1) Divide the 211 time-series into 36 groups according to 36 cases. 
(2) Divide the 211 time-series into 12 groups according to 2 states and 6 channels.

(3) Divide them into 2 groups according to 2 states.

4) Analyze time-energy series after grouping by using descriptive statistical analysis, in which the mean and median were computed to evaluate the central tendency and the standard deviation and quantile were computed to evaluate the dispersion of data set, and visualize static results.

\section{Results and Discussion}

In this part, 36 cases of coma and quasi-brain-death patients' EEG were analyzed by Dynamic 2T-EMD firstly, then a total of 216 time-energy series (36 cases $\times 6$ channels) of dynamic energy data were obtained, in which there were 211 valid time-energy series since 5 channels of EEG data from 3 patients were invalid. And we grouped through 3 grouping ways proposed above and evaluated them by using descriptive statistical analysis. Finally results were visualized.

Results for 36 cases of EEG data. In this part, 211 time-energy series were grouped into 36 set of data based on cases. The mean, median, standard deviation and quantile were computed for every case for average channel. As shown in figure 1(a), it is intuitively observed that the mean and median of EEG energy for coma case is higher than that for quasi-brain-death case for average channel. Here the mean and median energy fluctuation range of coma cases and quasi-brain-death cases are $1.10 \times 10^{4} \sim 6.66 \times 10^{4}, 1.01 \times 10^{4} \sim 6.22 \times 10^{4}$ which are higher than $1.00 \times 10^{4}$, and $2.48 \times 10^{3} \sim 9.13 \times 10^{3}, 2.40 \times 10^{3} \sim 8.86 \times 10^{3}$ which are no higher than $1.00 \times 10^{4}$. And it is also shown that the variance range of standard deviation of coma cases is wider than that of quasi-brain-death cases from figure 1 (b) and figure 1 (c).

Results based on 2 states -6 channels. In order to get characteristics of EEG energy for coma group and quasi-brain-death group in different channels of this batch of EEG energy data, 211 time-energy series were grouped into 12 set of data based on 2 states and 6 channels. Mean, median, standard deviation and quantile were also calculated for the 12 set of data. As shown in figure 2(a), (b) and (c), it is obviously observed that the mean and median of coma group is higher than that of quasi-brain-death group for every channel, and the range of energy change of every channel for coma group is wider than that for quasi-brain-death group. Moreover, values of mean, median and fluctuation percent between mean and median value of every channel for 2 groups were shown in table I and table II, in which percent is defined as percent $=($ Mean-Median $) /$ Mean, it is used to measure the difference between mean and median value. In general, it is shown that the percent for quasi-brain-death group is higher compared with the percent for coma group.

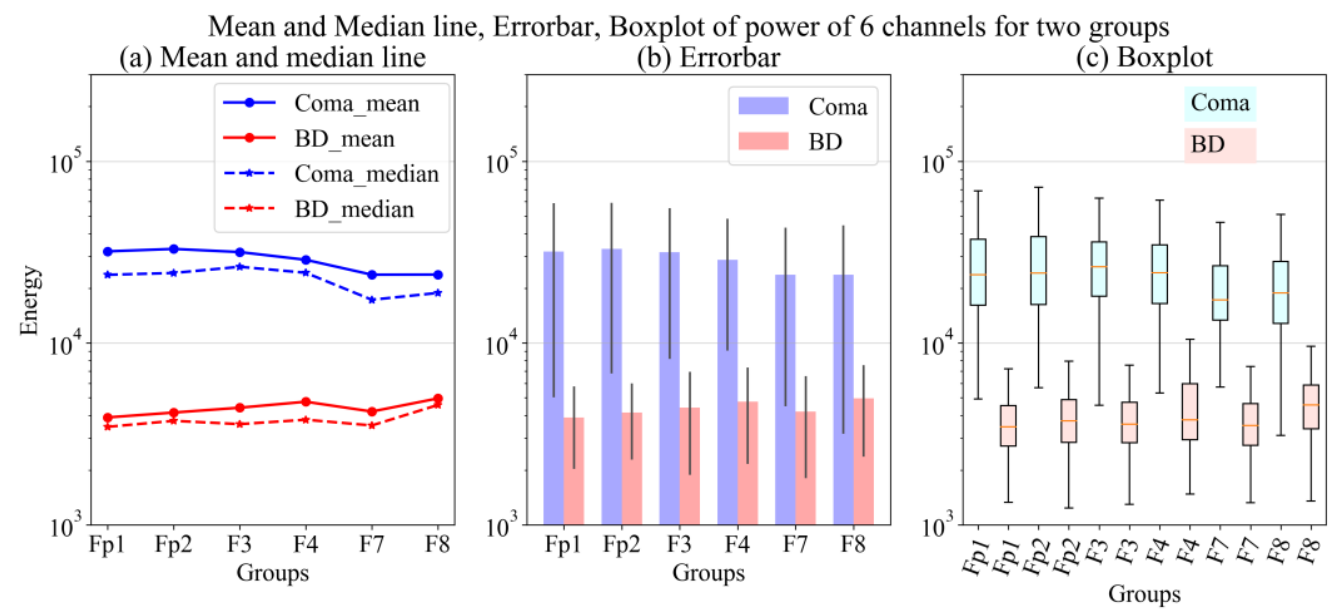

Figure 2. The statistical analysis results of EEG energy for coma group and quasi-brain-death group at each channel.

Table I. The mean, median and percent of EEG energy for coma group every channel.

\begin{tabular}{ccccccc}
\hline \multirow{2}{*}{ Coma } & \multicolumn{7}{c}{ Channel } \\
\cline { 2 - 7 } & Fp1 & Fp2 & F3 & F4 & F7 & F8 \\
\hline Mean & 31946 & 32951 & 31666 & 28708 & 23772 & 23804 \\
Median & 23735 & 24299 & 26274 & 24351 & 17303 & 18872 \\
Percent & $25.70 \%$ & $26.26 \%$ & $17.03 \%$ & $15.18 \%$ & $27.21 \%$ & $20.72 \%$ \\
\hline
\end{tabular}


Table II. The mean, median and percent of EEG energy for quasi-brain-death group at every channel

\begin{tabular}{ccccccc}
\hline \multirow{2}{*}{$\begin{array}{c}\text { Brain- } \\
\text { death }\end{array}$} & \multicolumn{7}{c}{ Channel } \\
\cline { 2 - 7 } & Fp1 & Fp2 & F3 & F4 & F7 & F8 \\
\hline Mean & 3899 & 4147 & 4410 & 4756 & 4201 & 4962 \\
Median & 3461 & 3734 & 3581 & 3787 & 3521 & 4564 \\
Percent & $11.23 \%$ & $9.96 \%$ & $18.80 \%$ & $20.37 \%$ & $16.19 \%$ & $8.02 \%$ \\
\hline
\end{tabular}

Results based on 2 states. Here the characteristics of energy for coma group and quasi-brain-death group for average channel were shown in figure 3 , in which there were 114 time-energy series (19 cases $\times 6$ channels - 5 invalid series) for coma group and 102 time-energy series ( 17 cases $\times 6$ channels) for quasibrain-death group. As can be seen from figure 3(a) and figure 3(b), the distribution of energy data for coma group is intuitively higher than that for quasi-brain-death group, in which the mean of both group are $2.88 \times 10^{4}$ and $4.40 \times 10^{3}$, and the median of both group are $2.24 \times 10^{4}$ and $3.73 \times 10^{3}$ respectively. Furthermore, the distribution of energy for coma group is obviously wider than that for quasi-brain-death group from figure 3(b) and figure3(c).
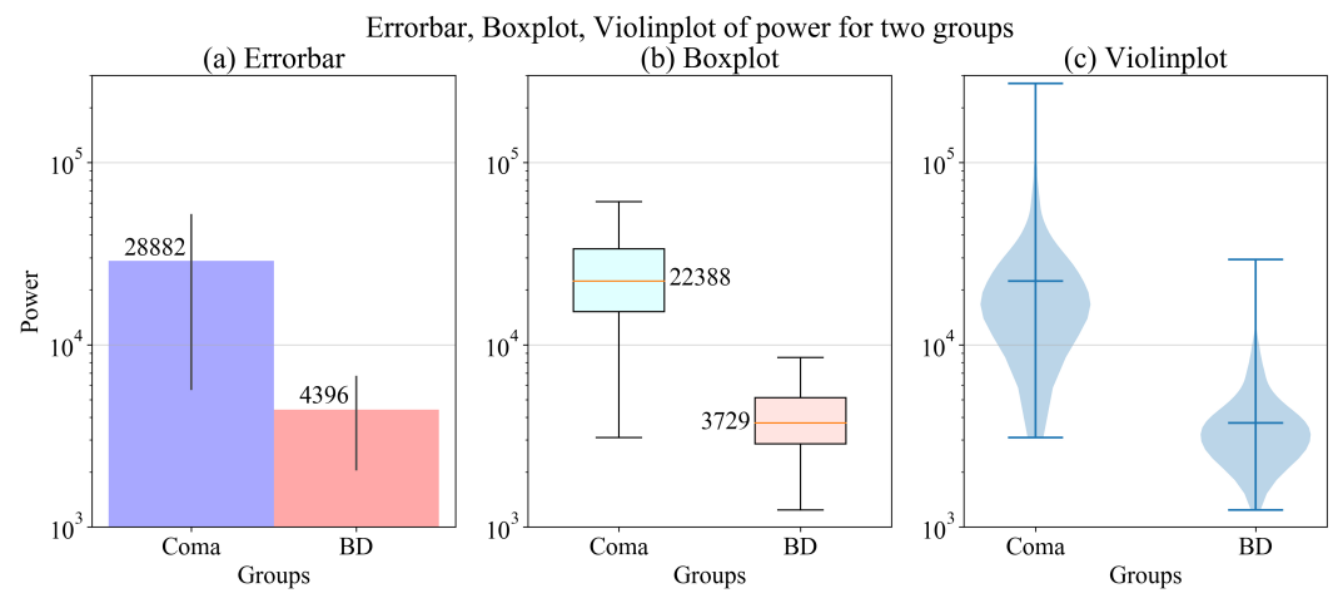

Figure 3. The error bar, violin plot and box plot of coma group and quasi-brain-death group for average channel.

Discussion. Results indicate that the EEG energy distribution in coma state is obviously higher than that in quasi-brain-death state from statistical and visual analysis aspect. There are 2 aspects discussed.

Firstly, in order to reduce the effect of extremum on the mean, it is necessary to combine the mean and median to discuss comprehensively the central tendency of 36 cases of EEG energy which obtained by using Dynamic 2T-EMD. If the mean and median differ too much, it is possible that the extrema affects the results, and the median is used as the measure to describe the central tendency in data set. As can be seen from table I and table II, there is a larger difference between the mean and median at each channel for coma group and quasi-brain-death group respectively. So here the median is used as measure to illustrate the central tendency. And as shown in figure 1(a), figure 2(a) and figure 3(a), the median of EEG energy in quasi-braindeath state is significantly lower that in coma state, in which the median of EEG energy in quasi-brain-death state is obvious lower than $1.00 \times 10^{4}$, while that in coma state is higher than $1.00 \times 10^{4}$.

Secondly, standard deviation and percentile are introduced to measure the dispersion of data set. As shown in figure 1(b), figure 1(c), figure 2(b), figure 2(c), figure 3(b) and figure 3(c), EEG energy data set in coma state is more discrete while EEG energy data set in quasi-brain-death is more concentrate.

Generally, the higher EEG energy is, the more obvious brain activity is. So we can infer that there is brain activity in coma subjects, while quasi-brain-death subjects have almost no brain activity.

\section{Conclusions}

In this paper, EEG energy from 19 cases of coma EEG and 17 cases of quasi-brain-death EEG were analyzed by descriptive statistical analysis, in which EEG energy was calculated by applying Dynamic 2T- 
EMD. Specifically, 211 time-energy series were grouped by according to three grouping standards firstly, respectively different cases, different channels and different state. And then mean, median, standard deviation and percentile values were computed separately for each group. Finally results obtained were visualized. Results show that compared with EEG energy for quasi-brain-death cases, the EEG energy for coma cases are higher and more discrete. Here the EEG energy in coma state is higher than $1.00 \times 10^{4}$, and EEG energy in quasi-brain-death state is lower than $1.00 \times 10^{4}$. This will be useful to study further features of coma and quasibrain-death patients' EEG.

\section{Acknowledgments}

This work was partly supported by KAKENHI (25420417, 15H04002 and 17K00326).

\section{References}

[1] Cao J.: "Analysis of the quasi-brain-death determination EEG data based on a robust ICA approach". Lecture Notes of in Artificial Intelligence, Springer, pp. 1240-1247, 2006.

[2] Z. Chen, J. Cao, Y. Cao, et al.: "Qualitative evaluation and quantitative EEG analysis in brain death diagnosis for adults: An empirical study", Cognitive Neurodynamics, Springer, Vol. 2, No. 3, pp. 257-271, 2008.

[3] N. Huang, Z. Shen, S. Long, M. Wu, H. Shih, Q. Zheng, N. Yen, C. Tung, and H. Liu: "The empirical mode decomposition and Hilbert spectrum for non-linear and non-stationary time series analysis", Proceedings of the Royal Society of London, A 454, pp. 903-995, 1998.

[4] Q. Shi, J. Cao, T. Tanaka, R. Wang, and H. Zhu.: "EEG data analysis based on EMD for coma and quasibrain-death patient", Journal of Experimental and Theoretical Artificial Intelligence, Vol. 23, No. 1, pp. 97110, 2011.

[5] Yao Miao, Dongsheng Wang, Gaochao Cui, Li Zhu and Jianting Cao: "Analyzing patients' EEG energy for brain death determination based on Dynamic 2T-EMD". International Journal of Computers \& Technology. Vol. 16, pp. 717-720 (2017).

[6] Julien Fleureau, Jean-Claude Nunes, Amar Kachenoura, Laurent Albera, and Lotfi Senhadji: "Turning Tangent Empirical Mode Decomposition: A Framework for Mono- and Multivariate Signals", IEEE Trans Signal Process, Vol. 59, No. 3, pp. 1309-1316, 2011.

[7] Cao J., Chen Z.: "Advanced EEG signal processing in brain death diagnosis", In: Signal Processing Techniques for Knowledge Extraction and Information Fusion, Springer, pp. 275-298, 2008.

[8] Mann, Prem S.: Introductory Statistics (2nd ed.), Wiley, ISBN 0-471-31009-3, 1995. 